Article

\title{
Is Previous eGFR a Reliable Risk Factor for COVID-19 Death? Single Centre Analysis in Chronic Kidney Disease Patients in Northern Italy
}

\author{
Francesca Martino ${ }^{1, * \mathbb{D}}$, Giulia Fanton ${ }^{2}$, Fiammetta Zanetti ${ }^{2}$, Michela Pascarella ${ }^{3}$ and Giacomo Novara 4 (D) \\ 1 UO Nephrology, Dialysis and Kidney Transplant, San Bortolo Hospital, 36100 Vicenza, Italy \\ 2 International Renal Research Institute Vicenza, 36100 Vicenza, Italy; fntngiulia@gmail.com (G.F.); \\ zanetti.fiammetta@gmail.com (F.Z.) \\ 3 Department of Virology and Microbiology, San Bortolo Hospital, 36100 Vicenza, Italy; \\ michela.pascarella@aulss8.veneto.it \\ 4 Department of Surgery, Oncology, and Gastroenterology, Urology Clinic University of Padua, \\ 35100 Padua, Italy; giacomonovara@gmail.com \\ * Correspondence: francesca.martino.k@gmail.com; Tel.: +39-444-753-650
}

Citation: Martino, F.; Fanton, G.; Zanetti, F.; Pascarella, M.; Novara, G. Is Previous eGFR a Reliable Risk Factor for COVID-19 Death? Single Centre Analysis in Chronic Kidney Disease Patients in Northern Italy. BioMed 2022, 2, 82-87. https:// doi.org/10.3390/biomed2010008

Academic Editor: Wolfgang Graier

Received: 10 January 2022

Accepted: 3 February 2022

Published: 8 February 2022

Publisher's Note: MDPI stays neutral with regard to jurisdictional claims in published maps and institutional affiliations.

Copyright: (C) 2022 by the authors. Licensee MDPI, Basel, Switzerland. This article is an open access article distributed under the terms and conditions of the Creative Commons Attribution (CC BY) license (https:// creativecommons.org/licenses/by/ $4.0 /)$.

\begin{abstract}
COVID-19 remains a major world health problem, and its clinical manifestations can vary from an oligosymptomatic form to severe pulmonary infection, which can require invasive ventilation and is strictly related to death. Identifying risk factors for adverse outcomes is essential for performing adequate care and contrasting high mortality. Chronic kidney disease (CKD) is a widespread comorbidity and is a known risk factor for death during SARS-CoV-2 infection. The present study evaluates the death risk assessment during the COVID-19 pandemic in (CKD) patients, considering the baseline value of an estimated glomerular filtration rate (eGFR) and other possible risk factors. We retrospectively assessed the mortality risk in 150 patients with COVID-19 between 1 October and 31 December 2020. We evaluated eGFR, haemoglobin, albumin, uric acid, cholesterol, triglycerides, and significant risk factors, such as diabetes mellitus and cardiovascular disease in every patient. We had 53 deaths (35.3\%) during the observational period, significantly related to age, eGFR, albumin, and baseline nephropathy. In the multivariable analysis, only baseline eGFR and age were independent predictors of death during SARS-CoV-2 infection, with an OR equal to 0.96 and 1.067 , respectively. In conclusion, by our analysis, age, and the baseline eGFR were the only reliable predictors of death during COVID-19 in CKD patients.
\end{abstract}

Keywords: COVID-19; chronic kidney disease; eGFR; mortality risk

\section{Introduction}

Coronavirus disease (COVID-19) is still a major world health problem, considering more than 280 million subjects had SARS-CoV-2 infection, of whom more than five million died [1]. Predictive factors identification remains essential to identify patients with a higher risk of severe manifestations and mortality.

Chronic kidney disease (CKD) is a widespread morbidity with a global mean prevalence of around 13\% [2], and it is a progressive disease, classified in five stages of severity by the estimation of glomerular filtration rate (eGFR) [3]. Usually, CKD patients are asymptomatic. Only stage V CKD patients (eGFR under $15 \mathrm{~mL} / \mathrm{min} / 1.73 \mathrm{~m}^{2}$ ) can present peculiar symptoms (nausea, vomiting, itch, and asthenia) and biochemical impairment (sodium and potassium impairment, calcium, and phosphate impairment, anaemia, metabolic acidosis), which can require replacement therapy, such as haemodialysis, peritoneal dialysis, and kidney transplantation. Furthermore, CKD is considered the most prevalent risk factor [4] for developing an adverse outcome, severe pulmonary infection, and death during the SARS-CoV-2 pandemic. Specifically, a meta-analysis of over 3.5 million patients showed 
an OR for death as high as 5.81 and 8.69 in the all-age and $<70$ years old CKD patients, respectively [5]. Furthermore, often CKD is associated with diabetes mellitus and cardiovascular disease, which are other prevalent risk factors for death and adverse outcomes during a SARS-CoV-2 infection [5]. Finally, CKD is related to other conditions such as ageing, hypertension, and malnutrition [6-9], which usually affects CKD patients' survival and could affect the outcome during COVID-19.

Ideally, CKD severity and associated comorbidities could be associated with a different susceptibility of CKD patients to SARS-CoV-2 infection. Currently, few studies have evaluated the impact of CKD severity on COVID-19 outcomes. The present study aims to assess if basal eGFR is a reliable risk factor for predicting adverse results during the COVID19 pandemic. Furthermore, we aim to evaluate the possible role of the other conditions during SARS-CoV-2 infection in CKD patients.

\section{Materials and Methods}

We performed a retrospective study in all stages of CKD patients who had COVID-19. Every patient was associated with a unique code (the first three letters of the surname, first three letters of the first name, followed by 1 for woman and 2 for man and six numbers for the date of birthdate, and the last three characters of the national health care code) to permit the identification of medical records and save patient privacy.

The study was carried out at the microbiology department of San Bortolo Hospital, Vicenza, Italy. All adult patients who had COVID-19 from 1 October to 31 December 2020 were eligible for the study. We randomly selected 30 patients, who belonged to $5 \mathrm{CKD}$ stages: I-II (reference, eGFR $>61 \mathrm{~mL} / \mathrm{min} / 1.73 \mathrm{~m}^{2}$ ), stage IIIa (eGFR $60-46 \mathrm{~mL} / \mathrm{min} / 1.73 \mathrm{~m}^{2}$ ), IIIb (eGFR $45-31 \mathrm{~mL} / \mathrm{min} / 1.73 \mathrm{~m}^{2}$ ), stage IV (eGFR $30-16 \mathrm{~mL} / \mathrm{min} / 1.73 \mathrm{~m}^{2}$ ), and V (eGFR $<15 \mathrm{~mL} / \mathrm{min} / 1.73 \mathrm{~m}^{2}$ ). The exclusion criteria were immunosuppression therapy, poor prognosis before a SARS-CoV-2 infection, and a previous SARS-CoV-2 positivity.

The detection of SARS-CoV-2 nucleic acids was performed on the Cobas 6800 reverse transcription-polymerase chain reaction (RT-PCR) system (Roche Diagnostic GmbH, Mannheim, Germany) from a nasopharyngeal swab.

In each patient, we evaluated the following baseline parameters: patient's characteristics (age, gender, type of outpatient clinic, and baseline nephropathy), comorbidity (cardiovascular disease, diabetes mellitus, liver disease, neoplasms, vascular disease), kidney function by an estimated glomerular filtration rate (eGFR) evaluated by the CKD-EPI formula 2009, haemoglobin $(\mathrm{g} / \mathrm{L})$, albumin $(\mathrm{g} / \mathrm{dL})$, cholesterol $(\mathrm{mg} / \mathrm{dL})$, triglycerides $(\mathrm{mg} / \mathrm{dL})$, and uric acid (mg/dL) levels.

According to the clinical notices, we recorded data available from one to six months before a SARS-CoV-2 infection.

Furthermore, for each patient, we evaluated admittance to a high-intensity care unit, where invasive mechanical ventilation or bi-level positive airway pressure, continuous positive airway pressure, and high-flow nasal cannula oxygen therapy was provided [10]; admittance to a medium-intensity care ward, where non-invasive respiratory support, such as a nasal cannula, simple mask, non-rebreather, or Venturi mask was provided [11]. We focused our analysis on mortality due to COVID-19 as a primary outcome.

\section{Statistical Analysis}

All continuous variables were reported as mean and standard deviation (SD) or median and interquartile range (IQR), as appropriate; categorical variables were described by number and percentage. The Shapiro-Wilk test tested all variables to evaluate the normal distribution. Student T, Mann-Whitney U, and chi-square tests were used to compare continuous and categorical variables, as appropriate. Univariable and multivariable logistic regression was used to evaluate the association of the covariates to death by the Odds Ratio (OR). Specifically, all the covariates with a $p$-value $<0.1$ in univariable analysis were included in the multivariable model. All reported $p$-values were two-sided, and the 
statistical significance was set at $p<0.05$. Statistical analysis was performed with IBM SPSS version 27 (SPSS Inc., Armonk, NY, USA).

We evaluated the sample size using the OpenEpi calculator (www.OpenEpi.com) on the basis of the recent report by Carlson et al. [12] comparing the death prevalence in normal kidney function to the prevalence reported for stage IIIa, IIIb, IV, and V CKD by the Kelsey formula with $80 \%$ power. Specifically, the estimated sample size was 26 subjects for stage IIIa (eGFR $46-60 \mathrm{~mL} / \mathrm{min} / 1.73 \mathrm{~m}^{2}$ ), 17 subjects for stage IIIlb (eGFR $31-45 \mathrm{~mL} / \mathrm{min} / 1.73 \mathrm{~m}^{2}$ ) and 15 subjects for stage IV (eGFR $16-30 \mathrm{~mL} / \mathrm{min} / 1.73 \mathrm{~m}^{2}$ ). Considering the possible covariates for multivariable analysis, we decided to enrol 150 patients (30 patients as the reference group without kidney damage and 30 for each stage: IIIa, IIIlb, IV, V).

\section{Results}

The characteristics of the 150 analysed patients are summarised in Table 1. During three months of observation, $16(10.6 \%)$ were admitted into high-intensity care wards, $24(16 \%)$ into medium-intensity care wards, and $5(3.3 \%)$ into low-intensity care wards. On the whole, 53 patients (34.1\%) died for COVID-19, of whom $8(15 \%)$ died at home, and $45(85 \%)$ died after hospital admission. The patients who died were significantly older, with a worse baseline kidney function evaluated by eGFR and lower baseline albumin levels (Table 1). Specifically, baseline eGFR values were significantly lower in those patients who died 22 (IQR:12-42) $\mathrm{mL} / \mathrm{min} / 1.73 \mathrm{~m}^{2}$ vs. 44 (IQR:22-59) $\mathrm{mL} / \mathrm{min} / 1.73 \mathrm{~m}^{2}$ in those patients who survived; $p<0.001)$

Table 1. Characteristic of all populations, and according for death during COVID-19.

\begin{tabular}{|c|c|c|c|c|}
\hline Variable & All Population (150) & COVID-19 Survivals (97) & COVID-19 Deaths (53) & $p$ Values \\
\hline Males (\%) & 52.2 & 49.5 & 57.4 & 0.4 \\
\hline Diabetes mellitus (\%) & 39.9 & 34.1 & 51.1 & 0.1 \\
\hline Cardiovascular disease (\%) & 56.5 & 50.5 & 68.1 & 0.1 \\
\hline Neoplasm (\%) & 20.3 & 17.6 & 25.5 & 0.4 \\
\hline Liver disease (\%) & 7.2 & 9.9 & 8.5 & 0.7 \\
\hline Baseline nephropathy: & 13 & 8.8 & 21.3 & $<0.001 *$ \\
\hline Diabetic nephropathy (\%) & 10.1 & 13.1 & 4.3 & \\
\hline Glomerulonephritis (\%) & 31.2 & 39.6 & 14.9 & \\
\hline Hypertensive nephropathy (\%) & 2.2 & 3.3 & 0 & \\
\hline Kidney stone (\%) & 5.1 & 5.5 & 4.3 & \\
\hline $\mathrm{PKD}^{\mathrm{a}}(\%)$ & 4.3 & 4.4 & 4.3 & \\
\hline Chronic Obstructive (\%) & 4.3 & 3.3 & 6.4 & \\
\hline Drug related $(\%)$ & 13 & 9.9 & 19.1 & \\
\hline Vascular disease $(\%)$ & 5.8 & 6.6 & 4.2 & \\
\hline Others $(\%)$ & 10.9 & 5.5 & 21.3 & \\
\hline Unknown (\%) & 13 & 8.8 & 21.3 & \\
\hline Age (years) & $(67-85)$ & $(62-83)$ & $(75-88)$ & $<0.001$ * \\
\hline $\mathrm{eGFR}\left(\mathrm{mL} / \mathrm{min} / 1.73 \mathrm{~m}^{2}\right)$ & $(15.7-52.2)$ & $(62-83)$ & $(12-42)$ & $<0.001 *$ \\
\hline Haemoglobin $(\mathrm{g} / \mathrm{L})$ & \pm 21.8 & \pm 24.5 & \pm 15 & 0.3 \\
\hline Albumin (g/dL) & $(3.6-4.3)$ & $(3.9-4.3)$ & $(3.5-4.1)$ & $0.02 *$ \\
\hline Cholesterol (mg/dL) & $(258-200.7)$ & $(125.5-203.5)$ & $(125-171)$ & 0.2 \\
\hline Triglycerides (mg/dL) & $(96.7-193)$ & $(87.7-192.2)$ & $(102-195)$ & 0.9 \\
\hline Uric Acid (mg/dL) & $(5.5-7.8)$ & $(5.25-7.2)$ & $(5.87-9.5)$ & 0.06 \\
\hline
\end{tabular}

${ }^{a}$ PKD $=$ Polycystic Kidney Disease. * Statistically significant. 
Table 2 summarises univariable analysis for the prediction of death. Baseline eGFR was significantly associated with death $(\mathrm{OR}=0.97 ; p<0.001)$, as well as older age $(\mathrm{OR}=1.051$, $p<0.001)$, and albumin level (OR 0.29; $p=0.04)$.

Table 2. Univariable analysis.

\begin{tabular}{lccc}
\hline \multicolumn{1}{c}{ Covariate } & OR & 95\% CI ${ }^{\wedge}$ of OR & $p$ Values \\
\hline Age (continuous) & 1.05 & $1.023-1.097$ & $<0.001^{*}$ \\
\hline Gender & 0.725 & $0.35-1.47$ & 0.4 \\
\hline Cardiovascular disease & 2.04 & $0.97-4.27$ & 0.06 \\
\hline Diabetes mellitus & 1.98 & $0.98-4.07$ & 0.06 \\
\hline Baseline eGFR (continuous) & 0.97 & $0.95-0.98$ & $<0.001^{*}$ \\
\hline Haemoglobin (continuous) & 0.98 & $0.95-1.002$ & 0.1 \\
\hline Albumin (continuous) & 0.294 & $0.093-0.93$ & $0.04{ }^{*}$ \\
\hline Cholesterol (continuous) & 0.994 & $0.98-1.003$ & 0.1 \\
\hline Triglycerides (continuous) & 0.999 & $0.99-1.005$ & 0.8 \\
\hline Uric acid (continuous) & 1.185 & $0.99-1.415$ & 0.1 \\
\hline${ }^{*}$ CI Confidence Interval ${ }^{*}$ Statistically significant. & &
\end{tabular}

Table 3 summarises multivariable analysis for the prediction of death. Finally, the age $(\mathrm{OR}=1.067 ; p=0.02)$ and baseline eGFR $(\mathrm{OR}=0.96 ; p=0.03)$ were independent predictors of death for COVID-19 in CKD patients.

Table 3. Multivariable analysis.

\begin{tabular}{lccc}
\hline \multicolumn{1}{c}{ Covariate } & OR & 95\% CI of $^{\text {OR }}$ & $p$ Values \\
\hline Age (continuous) & 1.067 & $1.01-1.128$ & $0.02{ }^{*}$ \\
\hline Cardiovascular disease & 0.65 & $0.146-2.95$ & 0.6 \\
\hline Diabetes mellitus & 1.34 & $0.37-4.82$ & 0.6 \\
\hline eGFR (continuous) & 0.96 & $0.92-0.99$ & 0.03 * \\
\hline Albumin (continuous) & 0.28 & $0.075-1.057$ & 0.1 \\
\hline
\end{tabular}

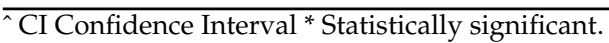

\section{Discussion}

We showed age and the baseline eGFR were the only reliable predictors of death during COVID-19 in CKD patients. Our results not only supported the previous studies, which showed how CKD is a risk factor for death during COVID-19 but showed the peculiar meaning of eGFR (OR 0.96) in positive CKD patients. Our data showed eGFR was a reliable risk factor addressed to death during COVID-19 in a cohort of patients with different stages of CKD and was followed by nephrology. Furthermore, we confirmed patient age as an independent predictor of death with SARS-CoV-2 infection (OR 1.067). In contrast, we did not observe any significant risk increment in diabetes mellitus and cardiovascular patients.

Our study showed the value of eGFR in the death risk assessment for the CKD population for the first time. Previous studies $(16,17,18)$ had shown how the presence of CKD or renal replacement therapy was a risk factor for death during COVID-19, but no studies addressed to assess the baseline value of eGFR in CKD patients from stage III to stage V. Only Carlson et al. (12) analysis reported the vulnerability to COVID-19 in CKD according to the five stages. They showed how the risk of death is directly associated with the increasing CKD stage. Our results moved in the same direction, but we showed the weight of every $\mathrm{mL} / \mathrm{min} / 1.73 \mathrm{~m}^{2}$ eGFR decrease on death risk in CKD patients. Furthermore, we considered baseline eGFR in a specific period from one to six months 
before COVID-19, whereas Carlson considered the eGFR until seven days before COVID-19 without any further details. Consequently, baseline eGFR can suffer from SARS-CoV-2 infection if the sample was obtained 7 days before the COVID-19 diagnosis or can suffer from the lack of reliability if the sample was obtained years before COVID-19.

Conversely, our series did not corroborate that diabetes mellitus and cardiovascular disease were independent predictors of death during COVID-19. Those results seem conflicting, with respect to other reports. We would like to underline some considerations about this discrepancy. Our study intended to assess the meaning of eGFR in COVID-19 mortality risk in CKD patients, so a relatively small number of cases could dilute the meaning of other conditions. Furthermore, diabetes mellitus and cardiovascular disease are usually higher prevalent comorbidities in CKD patients and this could cause their predictive value in the CKD population to decrease or even be lost. However, future studies need to explore this hypothesis.

Our results corroborated the previous results on the death rate estimated between 15 and $45 \%$ [13-16] and the prevalence of admission in intensive care units $[17,18]$ for CKD patients.

The present study had some limitations. First and foremost, the retrospective study design. Second, the study enrolled patients in a single centre. Third, all patients were followed in the nephrology department, especially in stages IV and V of CKD, which could mean a higher standard of care and prompt intervention, thus ideally affecting the primary outcome.

\section{Conclusions}

The present study corroborated previous reports showing how CKD is a significant risk factor for COVID-19 death and evaluated the value of eGFR in assessing death risk during COVID-19. Furthermore, it suggested how the other risk factors, such as diabetes mellitus and cardiovascular disease could change the impact in CKD patients.

Author Contributions: Conceptualization, F.M.; methodology, F.M.; software, G.F. and F.Z.; validation, all authors; formal analysis, F.M., G.F. and F.Z.; investigation, F.M., G.F. and F.Z.; resources, F.M., G.F. and F.Z.; data curation, G.F., F.Z. and M.P.; writing-original draft preparation, F.M. and G.N.; writing-review and editing, all authors.; visualization, all authors.; supervision, G.N.; project administration, F.M. All authors have read and agreed to the published version of the manuscript.

Funding: This research received no external funding.

Institutional Review Board Statement: The study was conducted in accordance with the Declaration of Helsinki. The ethical review and approval were waived for this study, and as required for the retrospective clinical investigation, we informed the local ethics committee.

Informed Consent Statement: Patient consent was waived because obtaining it would have made the conduct of the research impracticable, considering the lack of phone contact and the costs to locate the persons whose records were examined. Furthermore, subjects were not exposed to any risk by the irreversible anonymisation of data. The anonymisation process prevented any possible transmission of sensible data, saving subject privacy.

Data Availability Statement: Data available on request due to restrictions, e.g., privacy or ethics. The data presented in this study are available on request from the corresponding author.

Conflicts of Interest: The authors declare no conflict of interest.

\section{References}

1. WHO COVID-19 Dashboard. Available online: https:// covid19.who.int/ (accessed on 28 December 2021).

2. Hill, N.R.; Fatoba, S.T.; Oke, J.L.; Hirst, J.A.; O'Callaghan, C.A.; Lasserson, D.S.; Hobbs, F.D.R. Global Prevalence of Chronic Kidney Disease-A Systematic Review and Meta-Analysis. PLoS ONE 2016, 11, e0158765. [CrossRef] [PubMed]

3. Improving Global Outcomes (KDIGO) CKD Work Group. KDIGO 2012 Clinical Practice Guideline for the Evaluation and Management of Chronic Kidney Disease. Kidney inter. Suppl. 2013, 3, 1-150. [CrossRef] 
4. $\quad$ Clark, A.; Jit, M.; Warren-Gash, C.; Guthrie, B.; Wang, H.H.X.; Mercer, S.W.; Sanderson, C.; McKee, M.; Troeger, C.; Ong, K.L.; et al. Global, Regional, and National Estimates of the Population at Increased Risk of Severe COVID-19 Due to Underlying Health Conditions in 2020: A Modelling Study. Lancet Glob. Health 2020, 8, e1003-e1017. [CrossRef]

5. Cai, R.; Zhang, J.; Zhu, Y.; Liu, L.; Liu, Y.; He, Q. Mortality in Chronic Kidney Disease Patients with COVID-19: A Systematic Review and Meta-Analysis. Int. Urol. Nephrol. 2021, 53, 1623-1629. [CrossRef]

6. Guligowska, A.; Corsonello, A.; Pigłowska, M.; Roller-Wirnsberger, R.; Wirnsberger, G.; Ärnlöv, J.; Carlsson, A.C.; Tap, L.; Mattace-Raso, F.; Formiga, F.; et al. Association between Kidney Function, Nutritional Status and Anthropometric Measures in Older People. BMC Geriatr. 2020, 20, 366. [CrossRef] [PubMed]

7. Hamrahian, S.M.; Falkner, B. Hypertension in Chronic Kidney Disease BT-Hypertension: From Basic Research to Clinical Practice; Islam, M.S., Ed.; Springer International Publishing: Cham, Switzerland, 2017. [CrossRef]

8. Blackstone, E.; Morrison, M.; Roth, M.B. H2S Induces a Suspended Animation-like State in Mice. Science 2005, 308, 518. [CrossRef] [PubMed]

9. Al-Wahsh, H.; Lam, N.N.; Liu, P.; Quinn, R.R.; Fiocco, M.; Hemmelgarn, B.; Tangri, N.; Tonelli, M.; Ravani, P. Investigating the Relationship Between Age and Kidney Failure in Adults With Category 4 Chronic Kidney Disease. Can. J. Kidney Health Dis. 2020, 7, 2054358120966819. [CrossRef] [PubMed]

10. Bonnesen, B.; Jensen, J.-U.S.; Jeschke, K.N.; Mathioudakis, A.G.; Corlateanu, A.; Hansen, E.F.; Weinreich, U.M.; Hilberg, O.; Sivapalan, P. Management of COVID-19-Associated Acute Respiratory Failure with Alternatives to Invasive Mechanical Ventilation: High-Flow Oxygen, Continuous Positive Airway Pressure, and Noninvasive Ventilation. Diagnostics 2021, 11, 2259. [CrossRef] [PubMed]

11. Popowicz, P.; Leonard, K. Noninvasive Ventilation and Oxygenation Strategies. Surg. Clin. N. Am. 2022, 102, 149-157. [CrossRef] [PubMed]

12. Carlson, N.; Nelveg-Kristensen, K.-E.; Freese Ballegaard, E.; Feldt-Rasmussen, B.; Hornum, M.; Kamper, A.-L.; Gislason, G.; Torp-Pedersen, C. Increased Vulnerability to COVID-19 in Chronic Kidney Disease. J. Intern. Med. 2021, 290, 166-178. [CrossRef] [PubMed]

13. Mirijello, A.; Piscitelli, P.; de Matthaeis, A.; Inglese, M.; D’Errico, M.M.; Massa, V.; Greco, A.; Fontana, A.; Copetti, M.; Florio, L.; et al. Low EGFR Is a Strong Predictor of Worse Outcome in Hospitalized COVID-19 Patients. J. Clin. Med. 2021, 10, 5224. [CrossRef]

14. Wang, D.; Hu, B.; Hu, C.; Zhu, F.; Liu, X.; Zhang, J.; Wang, B.; Xiang, H.; Cheng, Z.; Xiong, Y.; et al. Clinical Characteristics of 138 Hospitalized Patients With 2019 Novel Coronavirus-Infected Pneumonia in Wuhan, China. JAMA 2020, 323, $1061-1069$. [CrossRef] [PubMed]

15. Guan, W.; Ni, Z.; Hu, Y.; Liang, W.; Ou, C.; He, J.; Liu, L.; Shan, H.; Lei, C.; Hui, D.S.C.; et al. Clinical Characteristics of Coronavirus Disease 2019 in China. N. Engl. J. Med. 2020, 382, 1708-1720. [CrossRef] [PubMed]

16. Russo, E.; Esposito, P.; Taramasso, L.; Magnasco, L.; Saio, M.; Briano, F.; Russo, C.; Dettori, S.; Vena, A.; Di Biagio, A.; et al. Kidney Disease and All-Cause Mortality in Patients with COVID-19 Hospitalized in Genoa, Northern Italy. J. Nephrol. 2021, 34, 173-183. [CrossRef] [PubMed]

17. Flythe, J.E.; Assimon, M.M.; Tugman, M.J.; Chang, E.H.; Gupta, S.; Shah, J.; Sosa, M.A.; Renaghan, A.D.; Melamed, M.L.; Wilson, F.P.; et al. Characteristics and Outcomes of Individuals With Pre-existing Kidney Disease and COVID-19 Admitted to Intensive Care Units in the United States. Am. J. Kidney Dis. 2021, 77, 190-203. [CrossRef] [PubMed]

18. Chung, E.Y.M.; Palmer, S.C.; Natale, P.; Krishnan, A.; Cooper, T.E.; Saglimbene, V.M.; Ruospo, M.; Au, E.; Jayanti, S.; Liang, A.; et al. Incidence and Outcomes of COVID-19 in People With CKD: A Systematic Review and Meta-analysis. Am. J. Kidney Dis. 2021, 78, 804-815. [CrossRef] [PubMed] 